\title{
A role for GABAergic interneuron diversity in circuit development and plasticity of the neonatal cerebral cortex
}

\author{
Simon J. B. Butt*, Jacqueline A. Stacey, Yayoi Teramoto, Cristiana Vagnoni \\ Department of Physiology, Anatomy \& Genetics, University of Oxford, South Parks Road, \\ Oxford, OX1 3QX, UK
}

* corresponding author. Email: simon.butt@dpag.ox.ac.uk

\begin{abstract}
GABAergic interneurons are a highly heterogeneous group of cells critical for the mature function and development of the neocortex. In terms of the latter, much attention has focused on the well-established role of parvalbumin (PV+)-expressing, fast spiking, basket cells in determining the critical period plasticity. However recent endeavours have started to shed the light on the contribution of other interneuron subtypes to early circuit formation and plasticity. Data that suggest that there are significant interactions between PV+ cells and other interneuron subtypes that regulate circuit development in rodents in the first postnatal week. Moreover a number of these early interactions are transient which points to an important, distinct role for interneuron diversity in setting up emergent neocortical processing.
\end{abstract}

\section{Highlights}

- Interneuron diversity is established at the moment of neurogenesis.

- All interneuron subtypes contribute to early neonatal circuit formation.

- The interaction between interneuron subtypes is likely important for plasticity.

- These early interneuron circuits can be distinct from those present in adults. 


\section{Introduction}

Locally-projecting GABAergic interneurons arise from diverse neurogenic niches in the ventral ganglionic eminences of the telencephalon before tangentially migrating to populate the entire rostral-caudal extent of the developing pallium. Over the last decade in vitro, transplant and developmental genetic approaches have determined that the fate of cortical interneurons is largely dependent on the temporal and spatial origin of the interneuron [1-7]. This suggests that the basic logic of the GABAergic system - the array of diverse subtypes critical to cognitive processing in the mature brain [8-11] - is established early in life; a time point at which the influence of GABA neurotransmission of post-synaptic neurons undergoes a change in signal[12, 13]. While research to date has focused primarily on the role of glutamatergic neurons in emerging neonatal circuits $[14,15]$, these do not act in exclusion, with GABAergic interneurons having an important role in sculpting and controlling early activity [16, 17]. This review focuses on recent evidence that suggests that interneuron diversity is important for defining circuits and network plasticity in the neonatal rodent brain. Dissections of these contributions are vital given the relationship between a number of these cells types and neurodevelopmental psychiatric disorders [18-20].

\section{A prolonged time window for early circuit formation}

In the mature neocortex GABAergic interneurons can at a basic level be broken down into three main populations defined by expression of the calcium-binding protein parvalbumin $(\mathrm{PV}+)$, the neuropeptide somatostatin (SST+) and the serotonin ionotropic receptor (5-HT $\left.\mathrm{H}_{3} \mathrm{R}\right)$ [21]. Two of these populations - SST+ and 5- $\mathrm{HT}_{3} \mathrm{R}$ subtypes - show a bias in distribution across the depth of the cortex; being preferentially found in infragranular and supragranular layers respectively [21-26]. This distribution likely reflects their respective temporal origin [27] with SST+ interneurons born early in the medial ganglionic eminence (MGE) [4] whereas 5- $\mathrm{HT}_{3} \mathrm{R}$ subtypes are born later from the caudal ganglionic eminence (CGE) [6, 28] (Figure 1). PV+ cells originate from the MGE and are born across a broad temporal window. Even in rodents, the protracted nature of neurogenesis and migration means that GABAergic interneurons are arriving in the dorsal pallium as early as embryonic day 12 (E12) [29] but with some cells not sorting into their final destination layers for at least another two weeks [27]. At the onset of circuit formation around birth, GABAergic interneurons interact with two early transient neuronal populations necessary for correct cortical development, Cajal-Retzius (CR) cells and the subplate (extensively reviewed in reference [16]). CR cells, receive dense GABAergic input [30] and in turn innervate GABAergic cells in early development with GABA receptor signalling a known promoter of apoptosis in CR cells once layer formation is complete around 
the second postnatal week [31]. The other early neuronal population, glutamatergic SP neurons, are embedded with neurons that express a range of GABAergic markers [17, 32] and that electrical stimulation and pharmacology experiments suggest provide both tonic and synaptic GABAergic control of SP output [33]. Histological analysis has revealed that the shifting profile of GABAergic SP neurons contain a large number of interneuron subtypes labelled by the 5-HT ${ }_{3} \mathrm{R}-\mathrm{GFP}$ transgenic [17] in contrast to adjacent neocortical layer 6. While this could be biased by late born CGE-derived migratory cells, the continued imbalance at later ages suggest that this profile is not transient and more likely due to a unique signalling requirements of SP.

Both CR and SP neurons are fundamental to early circuit formation and set in motion a neonatal time period over which there are significant changes in the nature of activity generated in the emergent layers of neocortex [34-36]. Such activity is characterized by spontaneous synchronized events that propagate through a variety of local and long range circuits across neocortex with the first GABA-dependent events, termed cortical giant depolarising potentials (cGDPs), first apparent midway through the first postnatal week [35]; a time point at which $\mathrm{GABA}_{\mathrm{A}}$ receptor antagonists also modulate columnar early gamma oscillations (EGOs) [36]. Evidence from hippocampus suggest that early-born cells from the MGE are critical to GDP generation [37, 38•], but the involvement and contribution of GABAergic interneuron subtypes to neocortical activity has not currently been determined per se. Regardless of which subtype it is likely that the complex dynamics of GABA reversal potential, cGDP activity [39] and in vivo EGOs [36] provide the ideal environment to promote active synapse formation and consolidation in the neonatal brain [40, 41••].

\section{Parvalbumin-positive basket cells - traditional mediators of neocortical circuit maturation and plasticity}

A number of studies have focused on the role of the MGE derived parvalbumin (PV+) basket cells, primarily because these represent the largest group of GABAergic interneuron, are found across all layers of neocortex (Figure 1c), and are readily distinguished in the mature brain by their fast action potentials and basket cell morphologies. These cells undergo two fairly distinct phases of development in neocortex. The second phase, about which most is known, is the classic example of sensory experience-dependent maturation of PV+ cells in visual cortex [42, 43]. This event occurs relatively late in circuit development and in response to multiple checks and balances ranging from control of transcription [44] to GABAergic activity [45-47]. However this is not the onset of PV+ interneuron function in neocortex with this cell type integrating within the first postnatal week into local circuits in visual [48]• and somatosensory 
cortex [49]•. This initial phase of synaptic integration appears to be largely governed by molecular cues [50, 51••] and is instructed by afferent PYRs in a class-specific manner [52] including translaminar feed-forward connections [49] at a time when the corresponding pyramidal cell (PYR) - PYR connections are mediated by NMDA receptor 'silent' synapses [53]. The targeting of PV+ interneurons by precise glutamatergic connections suggest that this class play an important role in the emergent circuit, binding together nascent PYR components, even though at this stage they are incapable of sustaining high frequency activity [54]. Moreover it appears that early integration and maturation of PV+ cells reaches a critical threshold to terminate early network activity and initiate the second, sensory experiencedependent phase [51••, 55-57]. This progressive increase in glutamatergic drive is matched by increasing GABAergic control of PV+ cells by other interneuron subtypes (Daniel Lyngholm, SB unpublished data), a further level of control that would enable dis-inhibition of PYRs and a window of opportunity for plasticity [58•].

\section{An emergent role for SST+ interneuron subtypes in early circuits.}

Somatostatin- $(\mathrm{SST}+)$ positive interneuron subtypes have been shown to target $\mathrm{PV}+$ cells in the mature neocortex [59] (Figure 2a). This group of diverse, primarily dendrite-targeting cell originate in the $N k x 2-1$-expressing ventricular zone of the ganglionic eminences early during interneuron neurogenesis. Key determinants of SST+ maturation and glutamatergic afferent input include the activity-dependent transcription factors Satb1 [60, 61] and Npas4 [62]•. Evidence from studies in hippocampus, have shown that a number of these early born cells occupy a distinct role in neonatal circuits as Hub cells co-ordinating activity across the neonatal circuit [37, 63]. As such, Hub cells are important for emergent synchrony in the early hippocampus, information flow and ultimately appropriate synaptic integration [64]. In neocortex synaptic integration of SST+ cells occurs early in the first postnatal week and is primarily dominated by local afferent connections irrespective of the layer location [49]. Of note are a population of Martinotti cell, located in infragranular layer 5b, that participate in early feed-forward signalling from the thalamus [65••, 66••] and have been shown to regulate thalamic engagement of PV+ interneurons [65••] and spiny stellate neurons in layer 4 of somatosensory cortex [66••]. These circuits are transient with the L5b-L4 connections coincident with the critical period plasticity in whisker somatosensory cortex [66••](Figure 2b,c). Intriguingly L5b GABAergic cells also innervate somatosensory cortex SP neurons in the first few postnatal days (Daniel Lyngholm, SB unpublished data) and develop connections onto layer 2/3 PYRs following the L4 critical period plasticity [49], coincident with the development of L4 to L2/3 circuit maturation [67] and a change in spike timing dependent 
plasticity rules in supragranular layers [68]. While SST+ interneurons undoubtedly regulate the timing of emergent activity, it is also apparent that GABA release can control glutamatergic synapse formation at the cellular level $[41 \bullet \bullet, 69]$. It is also worth noting in relation to this subtype that perturbing signalling from SST+ cells leads to compensation by other interneurons [66]; data which point to network level control over the amount of GABA signalling in neonatal cortex. Taken together these data suggest that SST+ cells, primarily located in infragranular layers that possess ascending axons that innervate superficial layers are well positioned to initiate and/or influence synaptic plasticity in the developing neocortex.

\section{Synaptic integration of $5 \mathrm{HT}_{3} \mathrm{R}$ interneurons is influenced by neurotransmitter activity}

The third major group of GABAergic interneuron are a highly diverse array of subtypes commonly identified by their expression of $5-\mathrm{HT}_{3} \mathrm{R}$, but that are also recognised by their expression of a wide variety of other markers, notably vasointestinal peptide (VIP), calretinin (CR), reeling (Re) and neuropeptide Y (NPY). The distinct, late origin of these cells in the CGE has made them tractable to a number of genetic/activity manipulation strategies that have resolved subtype-specific rules for the integration of these cells in neocortex [70, 71]. In brief, Re- and CR-positive cells types require normal levels of activity for their migration and integration into the early cortical circuit whereas VIP-positive cells require cell-autonomous expression of the transcription factor Prox1 to integrate fully into the PYR network. This suggests that the latter are perhaps more hard-wired into the neonatal circuit, in a manner independent of PYR activity. Unsurprisingly, cells from the CGE are susceptible to manipulation of embryonic serotonin levels with $\mathrm{Re}+$ interneurons failing to migrate correctly in the absence of $5-\mathrm{HT}_{3} \mathrm{R}$ [72]. Whereas VIP+ cell migration is disrupted following altered serotonin reuptake [73], albeit that the later manipulation also impacts on thalamic afferent development [74]. Intriguingly, a similar manipulation - dosing with the serotonin reuptake inhibitor fluoxetine - restores plasticity in adult visual cortex via a GABA-dependent mechanism [75].

\section{Are VIP+ interneurons and dis-inhibition of pyramidal cells involved in circuit formation and early plasticity?}

Of the various $5-\mathrm{HT}_{3} \mathrm{R}$ subtypes, VIP+ cells have attracted a lot of attention as they are targeted by cross-modal synaptic connections and could promote plasticity via an indirect mechanism, 
through their selective inhibition of SST+ cells [59, 76, 77] and resultant disinhibition of PYR cells (Figure 2a); a process that recent evidence suggests is dependent on experience-dependent genetic events [78••], sensory state $[79,80]$ and the complex interaction between VIP+ and SST+ cells [81]. Evidence for such a mechanism comes from Stryker and colleagues [82••] who demonstrated in a series of short monocular deprivation experiments that activation of VIP+ cells through locomotor activity could potentiate ocular dominance plasticity in binocular V1, and confirmed that this involved specific inhibition of SST+ cells. This avenue for kindling plasticity is distinct from a broader manipulation - transplantation of CGE-derived precursors, which was not able to induce ocular dominance plasticity [83•, 84•] in contrast to MGE grafts [83•, 85]. The inability of CGE transplants to trigger plasticity could arise from reduced integration of transplanted cells into the mature visual circuit [83•] and/or a bias toward $\mathrm{Re}+$ cells in the surviving grafted neurons [84•]. Finally, it is worth noting that both Re + and VIP+ cells also receive thalamic afferent input $[28,86]$, suggesting a potential role for this cell type in integrating sensory signals from the thalamus in supragranular layers, and thus able to further shape emergent cortical circuits. Given the relatively late integration of these interneurons into the neonatal circuits it is easier to envisage that such role emerges toward the end of the first postnatal week (CV, SJBB, unpublished data), after the end of the L4 critical period plasticity in somatosensory cortex.

\section{Early interneuron circuits are distinct from those in the adult}

Interneuron circuits change over the course of development to create necessary activity for circuit development and refinement [49•, 65••, 66••, 87]. Unsurprisingly evidence from in vivo sensory systems suggest that the function of early GABAergic signalling in cortical processing shifts over development and is distinct from that encountered in adults [88, 89]. However this should not be regarded as a two-stage process - neonate versus adult - with GABAergic circuits continuing to alter [90] and change in response to altered sensory input even in the mature brain [91-94]. Moreover, as better genetic tools are developed to parse apart interneuron diversity, it is becoming more and more evident that interneuron subtypes contribute to early circuit maturation and plasticity in a myriad of ways. The transient nature of a number of these early circuits $[49 \bullet, 65 \bullet \cdot, 66 \bullet$ ] suggest that GABAergic interneuron can act as a scaffold for the acquisition of normal sensory processing (Figure 3). Moreover that there is a carefully choreographed transition in early GABAergic circuits to ensure that information flow through cortical networks is appropriately regulate at each and every stage. 


\section{Figure legends}

\section{Figure 1. Origin and destination of GABAergic interneurons}

(a) Neocortical GABAergic interneuron originate from 2 main proliferative zones: the medial (MGE; blue) and caudal ganglionic eminences (CGE, green). Interneuron precursors tangentially migrate (black arrows) up into the ventral pallium where they integrate with locally-born pyramidal cells (grey triangles). LGE, lateral ganglionic eminence; OB, olfactory bulb. (b) The MGE gives rise to both parvalbumin- $(\mathrm{PV}+)$ and somatostatin- (SST+) expressing cells types. The former (dark blue) are born throughout the embryonic time window for MGE neurogenesis, whereas SST+ cells (light blue) are preferentially born during early time points. The CGE gives rise to the third main group of interneurons - defined by expression of $5-\mathrm{HT}_{3} \mathrm{R}$ (green) - these cells are born at later embryonic timepoints. (c) The timing of interneuron subtype neurogenesis reflects the distribution across the depth of the six-layered cortex. Information transfer through the canonical cortical circuit is depicted by the black arrows. The proportion of the 3 main interneuron subtypes for layers 2/3, 4 and 5 are shown to the right [22, 26, 49•]. PV+ cells (dark blue segment) are fairly evenly distributed, while SST+ cells (light blue) are found in deep, infragranular layers. 5- $\mathrm{HT}_{3} \mathrm{R}+$ subtypes (green), including vasointestinal peptide-expressing (VIP+) interneurons are primarily found in greater number in superficial, supragranular layers (layers 2/3). WM, white matter; Th, thalamic afferent input.

\section{Figure 2. Early interneuron circuits can be distinct from those reported in adults}

(a) Both main types of MGE-derived interneuron (blue) form synaptic connections onto pyramidal cells (PYR; grey) with PV+ cells also forming extensive chemical and gap junction coupled networks with other PV+ cells. SST+ cells form synapses onto PV+ cells and also reciprocal connections with VIP+ cells. As such both SST + and VIP+ cells can disinhibit PYRs (adapted from reference [59]). (b) In neonatal somatosensory cortex L5b SST+ interneurons form a reciprocal synaptic loop with L4 spiny stellate neurons (SSNs; red cell)[66]. This is the main source of inhibition onto SSNs in the absence of mature PV+ input. (c) Following the end of the critical period plasticity the L5b-L4 loop collapses and mature feed-forward inhibition of SSNs emerges $[55,57]$.

\section{Figure 3. Time line of major circuit events in neonatal mouse somatosensory cortex.}

A time line from the day of birth, postnatal day (P)0, until P16. Major window are shown with light grey bars, network events with medium grey and interneuron integration and circuits shown with dark grey. CPP, critical period plasticity; Th, thalamus; L4, layer 4; L2/3, layer 2/3. cENO, cortical early network oscillation [35]; cGDP, cortical giant depolarising potential; EGOs, early gamma oscillations [36]. 
References

1. Nery, S., G. Fishell, and J.G. Corbin, The caudal ganglionic eminence is a source of distinct cortical and subcortical cell populations. Nat Neurosci, 2002. 5(12): p. 127987.

2. $\quad \mathrm{Xu}, \mathrm{Q}$., et al., Origins of cortical interneuron subtypes. J Neurosci, 2004. 24(11): p. 2612-22.

3. Butt, S.J., et al., The temporal and spatial origins of cortical interneurons predict their physiological subtype. Neuron, 2005. 48(4): p. 591-604.

4. Miyoshi, G., et al., Physiologically distinct temporal cohorts of cortical interneurons arise from telencephalic Olig2-expressing precursors. J Neurosci, 2007. 27(29): p. 7786-98.

5. Fogarty, M., et al., Spatial genetic patterning of the embryonic neuroepithelium generates GABAergic interneuron diversity in the adult cortex. J Neurosci, 2007. 27(41): p. 10935-46.

6. Miyoshi, G., et al., Genetic fate mapping reveals that the caudal ganglionic eminence produces a large and diverse population of superficial cortical interneurons. J Neurosci, 2010. 30(5): p. 1582-94.

7. Gelman, D., et al., A wide diversity of cortical GABAergic interneurons derives from the embryonic preoptic area. J Neurosci, 2011. 31(46): p. 16570-80.

8. Markram, H., et al., Interneurons of the neocortical inhibitory system. Nat Rev Neurosci, 2004. 5(10): p. 793-807.

9. Klausberger, T. and P. Somogyi, Neuronal diversity and temporal dynamics: the unity of hippocampal circuit operations. Science, 2008. 321(5885): p. 53-7.

10. Kepecs, A. and G. Fishell, Interneuron cell types are fit to function. Nature, 2014. 505(7483): p. 318-26.

11. Tremblay, R., S. Lee, and B. Rudy, GABAergic Interneurons in the Neocortex: From Cellular Properties to Circuits. Neuron, 2016. 91(2): p. 260-92.

12. Ben-Ari, Y., et al., GABA: a pioneer transmitter that excites immature neurons and generates primitive oscillations. Physiol Rev, 2007. 87(4): p. 1215-84.

13. Owens, D.F. and A.R. Kriegstein, Is there more to GABA than synaptic inhibition? Nat Rev Neurosci, 2002. 3(9): p. 715-27.

14. Kanold, P.O. and H.J. Luhmann, The subplate and early cortical circuits. Annu Rev Neurosci, 2010. 33: p. 23-48.

15. Luhmann, H.J., et al., Spontaneous Neuronal Activity in Developing Neocortical Networks: From Single Cells to Large-Scale Interactions. Front Neural Circuits, 2016. 10: p. 40.

16. Luhmann, H.J., et al., Early GABAergic circuitry in the cerebral cortex. Curr Opin Neurobiol, 2014. 26: p. 72-8.

17. Qu, G.J., et al., Postnatal development of GABAergic interneurons in the neocortical subplate of mice. Neuroscience, 2016. 322: p. 78-93.

18. Marin, O., Interneuron dysfunction in psychiatric disorders. Nat Rev Neurosci, 2012. 13(2): p. 107-20.

19. Volk, D.W. and D.A. Lewis, Early developmental disturbances of cortical inhibitory neurons: contribution to cognitive deficits in schizophrenia. Schizophr Bull, 2014. 40(5): p. 952-7.

20. Le Magueresse, C. and H. Monyer, GABAergic interneurons shape the functional maturation of the cortex. Neuron, 2013. 77(3): p. 388-405.

21. Rudy, B., et al., Three groups of interneurons account for nearly $100 \%$ of neocortical GABAergic neurons. Dev Neurobiol, 2011. 71(1): p. 45-61.

22. Gonchar, Y., Q. Wang, and A. Burkhalter, Multiple distinct subtypes of GABAergic neurons in mouse visual cortex identified by triple immunostaining. Front Neuroanat, 2007. 1: p. 3. 
23. Kubota, Y., et al., Selective coexpression of multiple chemical markers defines discrete populations of neocortical GABAergic neurons. Cereb Cortex, 2011. 21(8): p. 180317.

24. Meyer, H.S., et al., Inhibitory interneurons in a cortical column form hot zones of inhibition in layers 2 and 5A. Proc Natl Acad Sci U S A, 2011. 108(40): p. 16807-12.

25. Pronneke, A., et al., Characterizing VIP Neurons in the Barrel Cortex of VIPcre/tdTomato Mice Reveals Layer-Specific Differences. Cereb Cortex, 2015. 25(12): p. 4854-68.

26. Xu, X., K.D. Roby, and E.M. Callaway, Immunochemical characterization of inhibitory mouse cortical neurons: three chemically distinct classes of inhibitory cells. J Comp Neurol, 2010. 518(3): p. 389-404.

27. Miyoshi, G. and G. Fishell, GABAergic interneuron lineages selectively sort into specific cortical layers during early postnatal development. Cereb Cortex, 2011. 21(4): p. 845-52.

28. Lee, S., et al., The largest group of superficial neocortical GABAergic interneurons expresses ionotropic serotonin receptors. J Neurosci, 2010. 30(50): p. 16796-808.

29. Del Rio, J.A., E. Soriano, and I. Ferrer, Development of GABA-immunoreactivity in the neocortex of the mouse. J Comp Neurol, 1992. 326(4): p. 501-26.

30. Radnikow, G., D. Feldmeyer, and J. Lubke, Axonal projection, input and output synapses, and synaptic physiology of Cajal-Retzius cells in the developing rat neocortex. J Neurosci, 2002. 22(16): p. 6908-19.

31. Blanquie, O., et al., NKCC1-Mediated GABAergic Signaling Promotes Postnatal Cell Death in Neocortical Cajal-Retzius Cells. Cereb Cortex, 2016.

32. Chun, J.J., M.J. Nakamura, and C.J. Shatz, Transient cells of the developing mammalian telencephalon are peptide-immunoreactive neurons. Nature, 1987. 325(6105): p. 617-20.

33. Hanganu, I.L., W. Kilb, and H.J. Luhmann, Functional synaptic projections onto subplate neurons in neonatal rat somatosensory cortex. J Neurosci, 2002. 22(16): p. 7165-76.

34. Dupont, E., et al., Rapid developmental switch in the mechanisms driving early cortical columnar networks. Nature, 2006. 439(7072): p. 79-83.

35. Allene, C., et al., Sequential generation of two distinct synapse-driven network patterns in developing neocortex. J Neurosci, 2008. 28(48): p. 12851-63.

36. Minlebaev, M., et al., Early gamma oscillations synchronize developing thalamus and cortex. Science, 2011. 334(6053): p. 226-9.

37. Picardo, M.A., et al., Pioneer GABA cells comprise a subpopulation of hub neurons in the developing hippocampus. Neuron, 2011. 71(4): p. 695-709.

38. Wester, J.C. and C.J. McBain, Interneurons Differentially Contribute to Spontaneous Network Activity in the Developing Hippocampus Dependent on Their Embryonic Lineage. J Neurosci, 2016. 36(9): p. 2646-62.

- A detailed and comprehensive study that uses optogenetics and electrophysiology - as well as morphological analysis to dissect the contribution of both MGE- and CGE-derived interneurons to early network activity in acute in vitro hippocampal slices.

39. Khalilov, I., et al., Dynamic Changes from Depolarizing to Hyperpolarizing GABAergic Actions during Giant Depolarizing Potentials in the Neonatal Rat Hippocampus. J Neurosci, 2015. 35(37): p. 12635-42.

40. Mohajerani, M.H., et al., Correlated network activity enhances synaptic efficacy via BDNF and the ERK pathway at immature CA3 CA1 connections in the hippocampus. Proc Natl Acad Sci U S A, 2007. 104(32): p. 13176-81.

41. Oh, W.C., et al., De novo synaptogenesis induced by GABA in the developing mouse cortex. Science, 2016. 353(6303): p. 1037-1040.

-. This paper shows how first how GABA neurotransmission can instruct circuit formation at the cellular level and further demonstrates that GABA release from somatostatin interneurons can cause synaptogenesis. 
42. Huang, Z.J., et al., BDNF regulates the maturation of inhibition and the critical period of plasticity in mouse visual cortex. Cell, 1999. 98(6): p. 739-55.

43. Hensch, T.K., Critical period plasticity in local cortical circuits. Nat Rev Neurosci, 2005. 6(11): p. 877-88.

44. Krishnan, K., et al., MeCP2 regulates the timing of critical period plasticity that shapes functional connectivity in primary visual cortex. Proc Natl Acad Sci U S A, 2015. 112(34): p. E4782-91.

45. Hensch, T.K., et al., Local GABA circuit control of experience-dependent plasticity in developing visual cortex. Science, 1998. 282(5393): p. 1504-8.

46. Hensch, T.K. and M.P. Stryker, Columnar architecture sculpted by GABA circuits in developing cat visual cortex. Science, 2004. 303(5664): p. 1678-81.

47. Deidda, G., et al., Early depolarizing GABA controls critical-period plasticity in the rat visual cortex. Nat Neurosci, 2015. 18(1): p. 87-96.

48. Pangratz-Fuehrer, S. and S. Hestrin, Synaptogenesis of electrical and GABAergic synapses of fast-spiking inhibitory neurons in the neocortex. J Neurosci, 2011. 31(30): p. 10767-75.

49. Anastasiades, P.G., et al., GABAergic interneurons form transient layer-specific circuits in early postnatal neocortex. Nat Commun, 2016. 7: p. 10584.

- In this paper the authors employ laser scanning photostimulation to map afferent and efferent connections of MGE-derived interneurons across the depth of neonatal cortex. As such this builds on the earlier work of Pangratz-Fuehrer and Hestrin (2011) to describe early interneuron synaptic connections, including a number of transient circuits.

50. Fazzari, P., et al., Control of cortical GABA circuitry development by Nrg1 and ErbB4 signalling. Nature, 2010. 464(7293): p. 1376-80.

51. Pelkey, K.A., et al., Pentraxins coordinate excitatory synapse maturation and circuit integration of parvalbumin interneurons. Neuron, 2015. 85(6): p. 1257-72.

-. This paper describes a molecular mechanism involved in the regulation of parvalbumin interneuron integration. Using an impressive array of techniques the authors show how pextraxins assist in the emergence of appropriate excitatory-inhibitory balance.

52. Ye, Z., et al., Instructing Perisomatic Inhibition by Direct Lineage Reprogramming of Neocortical Projection Neurons. Neuron, 2015. 88(3): p. 475-83.

53. Anastasiades, P.G. and S.J. Butt, A role for silent synapses in the development of the pathway from layer 2/3 to 5 pyramidal cells in the neocortex. J Neurosci, 2012. 32(38): p. 13085-99.

54. Doischer, D., et al., Postnatal differentiation of basket cells from slow to fast signaling devices. J Neurosci, 2008. 28(48): p. 12956-68.

55. Chittajallu, R. and J.T. Isaac, Emergence of cortical inhibition by coordinated sensorydriven plasticity at distinct synaptic loci. Nat Neurosci, 2010. 13(10): p. 1240-8.

56. Gu, Y., et al., Obligatory role for the immediate early gene NARP in critical period plasticity. Neuron, 2013. 79(2): p. 335-46.

57. Daw, M.I., M.C. Ashby, and J.T. Isaac, Coordinated developmental recruitment of latent fast spiking interneurons in layer IV barrel cortex. Nat Neurosci, 2007. 10(4): p. 453-61.

58. Kuhlman, S.J., et al., A disinhibitory microcircuit initiates critical-period plasticity in the visual cortex. Nature, 2013. 501(7468): p. 543-6.

- An elegant study that uses a variety of optical and chemogenetic approaches to understand the circuit basis, and specifically the role of parvalbumin interneuron activity, in controlling the critical period plasticity.

59. Pfeffer, C.K., et al., Inhibition of inhibition in visual cortex: the logic of connections between molecularly distinct interneurons. Nat Neurosci, 2013. 16(8): p. 1068-76.

60. Denaxa, M., et al., Maturation-promoting activity of SATB1 in MGE-derived cortical interneurons. Cell Rep, 2012. 2(5): p. 1351-62. 
61. Close, J., et al., Satb1 is an activity-modulated transcription factor required for the terminal differentiation and connectivity of medial ganglionic eminence-derived cortical interneurons. J Neurosci, 2012. 32(49): p. 17690-705.

62. Spiegel, I., et al., Npas4 regulates excitatory-inhibitory balance within neural circuits through cell-type-specific gene programs. Cell, 2014. 157(5): p. 1216-29.

- One of a series of extremely thorough studies from the same group, that have dissected the contribution of activity-dependent genes to interneuron integration and emergent function.

63. Bonifazi, P., et al., GABAergic hub neurons orchestrate synchrony in developing hippocampal networks. Science, 2009. 326(5958): p. 1419-24.

64. Cossart, R., Operational hub cells: a morpho-physiologically diverse class of GABAergic neurons united by a common function. Curr Opin Neurobiol, 2014. 26: p. 51-6.

65. Tuncdemir, S.N., et al., Early Somatostatin Interneuron Connectivity Mediates the Maturation of Deep Layer Cortical Circuits. Neuron, 2016. 89(3): p. 521-35.

66. Marques-Smith, A., et al., A Transient Translaminar GABAergic Interneuron Circuit Connects Thalamocortical Recipient Layers in Neonatal Somatosensory Cortex. Neuron, 2016. 89(3): p. 536-49.

- [65 and 66] These two papers investigate the synaptic integration and role of layer 5b somatostatin interneurons in neonatal cortex. Both identify transient translaminar synaptic connections and an involvement of this interneuron subtype in thalamocortical circuit maturation. This suggets that early-born somatostatin interneurons are key players in neocortical development.

67. Bureau, I., G.M. Shepherd, and K. Svoboda, Precise development of functional and anatomical columns in the neocortex. Neuron, 2004. 42(5): p. 789-801.

68. Itami, C. and F. Kimura, Developmental switch in spike timing-dependent plasticity at layers 4-2/3 in the rodent barrel cortex. J Neurosci, 2012. 32(43): p. 15000-11.

69. Wang, D.D. and A.R. Kriegstein, GABA regulates excitatory synapse formation in the neocortex via NMDA receptor activation. J Neurosci, 2008. 28(21): p. 5547-58.

70. De Marco Garcia, N.V., T. Karayannis, and G. Fishell, Neuronal activity is required for the development of specific cortical interneuron subtypes. Nature, 2011.472(7343): p. 351-5.

71. Miyoshi, G., et al., Prox1 Regulates the Subtype-Specific Development of Caudal Ganglionic Eminence-Derived GABAergic Cortical Interneurons. J Neurosci, 2015. 35(37): p. 12869-89.

72. Murthy, S., et al., Serotonin receptor $3 A$ controls interneuron migration into the neocortex. Nat Commun, 2014. 5: p. 5524.

73. Frazer, S., K. Otomo, and A. Dayer, Early-life serotonin dysregulation affects the migration and positioning of cortical interneuron subtypes. Transl Psychiatry, 2015. 5: p. e644.

74. Bonnin, A., et al., Serotonin modulates the response of embryonic thalamocortical axons to netrin-1. Nat Neurosci, 2007. 10(5): p. 588-97.

75. Maya Vetencourt, J.F., et al., The antidepressant fluoxetine restores plasticity in the adult visual cortex. Science, 2008. 320(5874): p. 385-8.

76. $\mathrm{Pi}, \mathrm{H} . J$. , et al., Cortical interneurons that specialize in disinhibitory control. Nature, 2013. 503(7477): p. 521-4.

77. Lee, S., et al., A disinhibitory circuit mediates motor integration in the somatosensory cortex. Nat Neurosci, 2013. 16(11): p. 1662-70.

78. Mardinly, A.R., et al., Sensory experience regulates cortical inhibition by inducing IGF1 in VIP neurons. Nature, 2016. 531(7594): p. 371-5.

-• CGE-derived interneurons - including VIP+ cells - arrive relatively late into the developing neocortex. The mechanism by which they integrate and start to exert their function is explored in this detailed paper. 
79. Neske, G.T. and B.W. Connors, Distinct Roles of SOM and VIP Interneurons during Cortical Up States. Front Neural Circuits, 2016. 10: p. 52.

80. Dipoppa, M., et al., Vision and locomotion shape the interactions between neuron types in mouse visual cortex. bioRxiv, 2016.

81. Karnani, M.M., et al., Cooperative Subnetworks of Molecularly Similar Interneurons in Mouse Neocortex. Neuron, 2016. 90(1): p. 86-100.

82. Fu, Y., et al., A cortical disinhibitory circuit for enhancing adult plasticity. Elife, 2015. 4: p. e05558.

- $\quad$ The authors use an optogenetic strategy to probe the contribution of both VIP and SST interneurons to visual cortical plasticity. They show that VIP inhibition of SST cells - and as a consequence disinhibition of V1 pyramidal cells - is involved in this process.

83. Davis, M.F., et al., Inhibitory Neuron Transplantation into Adult Visual Cortex Creates a New Critical Period that Rescues Impaired Vision. Neuron, 2015. 86(4): p. 10551066.

84. Larimer, P., et al., Caudal Ganglionic Eminence Precursor Transplants Disperse and Integrate as Lineage-Specific Interneurons but Do Not Induce Cortical Plasticity. Cell Rep, 2016. 16(5): p. 1391-404.

- [83 and 84] Two recent publications that probe whether or not transplantation of CGE-derived interneurons can also trigger plasticity in adult cortex.

85. Southwell, D.G., et al., Cortical plasticity induced by inhibitory neuron transplantation. Science, 2010. 327(5969): p. 1145-8.

86. De Marco Garcia, N.V., et al., Sensory inputs control the integration of neurogliaform interneurons into cortical circuits. Nat Neurosci, 2015. 18(3): p. 393-401.

87. Allene, C., et al., Dynamic changes in interneuron morphophysiological properties mark the maturation of hippocampal network activity. J Neurosci, 2012. 32(19): p. 6688-98.

88. Dorrn, A.L., et al., Developmental sensory experience balances cortical excitation and inhibition. Nature, 2010. 465(7300): p. 932-6.

89. Kuhlman, S.J., E. Tring, and J.T. Trachtenberg, Fast-spiking interneurons have an initial orientation bias that is lost with vision. Nat Neurosci, 2011. 14(9): p. 1121-3.

90. Jiang, X., et al., Principles of connectivity among morphologically defined cell types in adult neocortex. Science, 2015. 350(6264): p. aac9462.

91. Katzel, D. and G. Miesenbock, Experience-dependent rewiring of specific inhibitory connections in adult neocortex. PLoS Biol, 2014. 12(2): p. e1001798.

92. Chen, J.L., et al., Structural basis for the role of inhibition in facilitating adult brain plasticity. Nat Neurosci, 2011. 14(5): p. 587-94.

93. Keck, T., et al., Loss of sensory input causes rapid structural changes of inhibitory neurons in adult mouse visual cortex. Neuron, 2011. 71(5): p. 869-82.

94. Marik, S.A., et al., Axonal dynamics of excitatory and inhibitory neurons in somatosensory cortex. PLoS Biol, 2010. 8(6): p. e1000395. 
Figure 1

Butt et al.

(a)

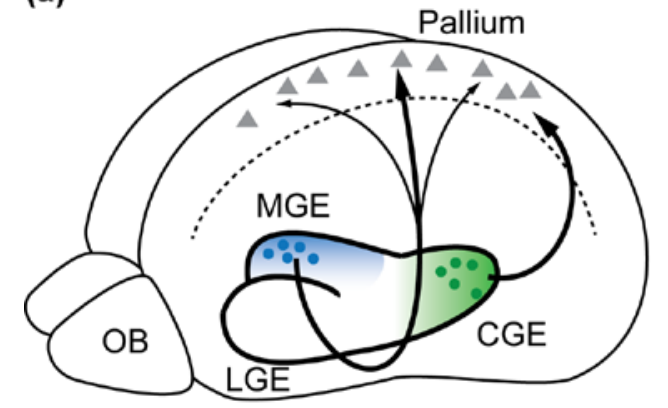

(b)

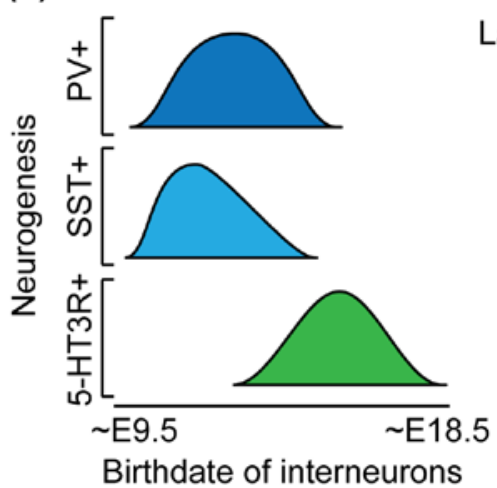

(c)

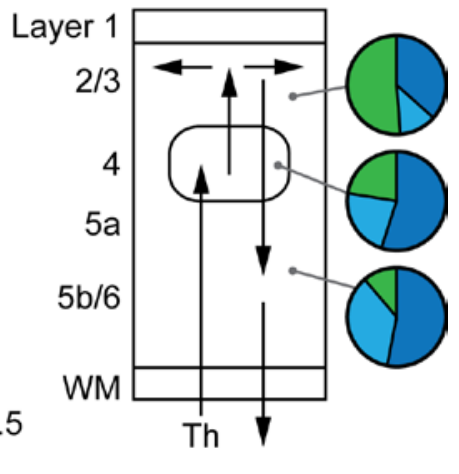


Figure 2

Butt et al.

(a)

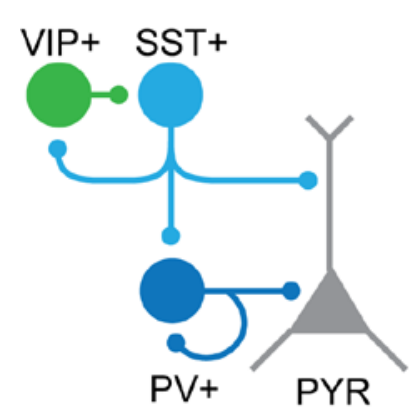

(b) Developing

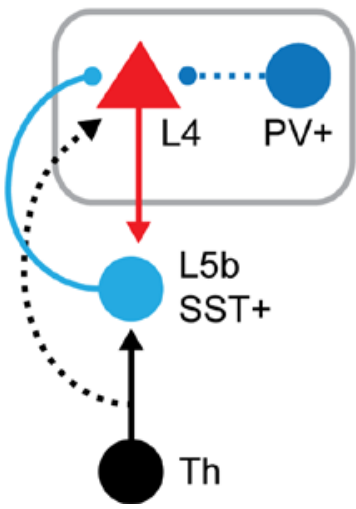

(c) Mature

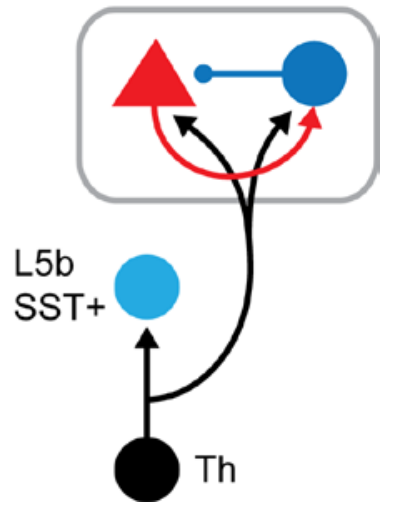


Figure 3

Butt et al.

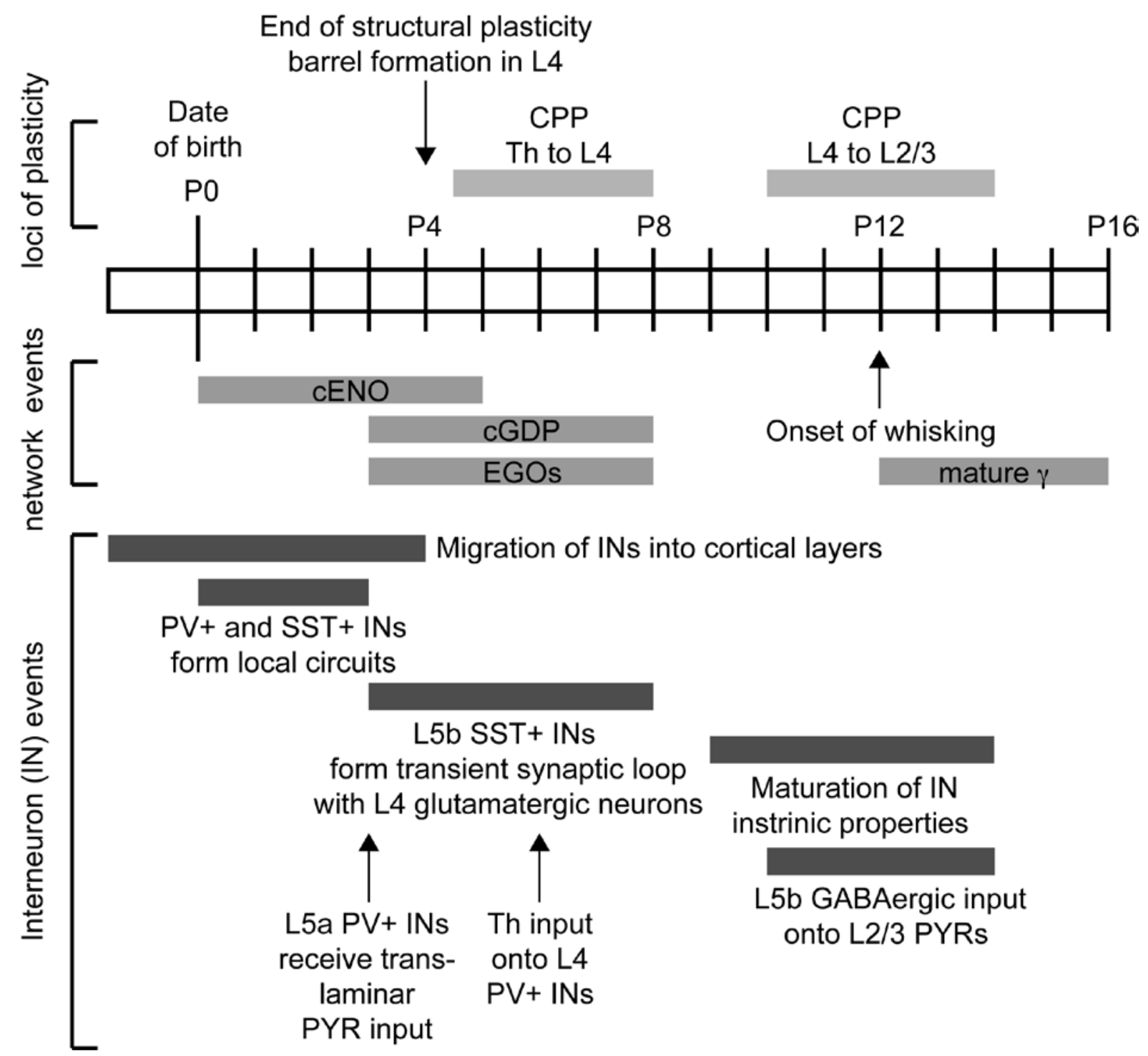

